\title{
Explorando uma Aplicação m-learning para Ensino de Vetores na Física do Ensino Médio
}

\author{
Eduardo Honorato ${ }^{1}$, Carlos Schocair ${ }^{1}$, João Quadros ${ }^{1}$, Rafael Castaneda ${ }^{1}$, \\ Jorge Soares $^{1,2}$, Renato Mauro ${ }^{1}$, Sérgio Duarte ${ }^{1}$, Eduardo Ogasawara ${ }^{1}$ \\ ${ }^{1}$ Centro Federal de Educação Tecnológica Celso Suckow da Fonseca - CEFET/RJ \\ ${ }^{2}$ Universidade do Estado do Rio de Janeiro - UERJ \\ \{schocair, jquadros, rcastaneda, jsoares, rmauro, sduart\}@cefet-rj.br \\ eogasawara@ieee.org
}

\begin{abstract}
The knowledge and understanding of vectors and vector quantities is extremely important to learn the motion of objects in physics at high school. Considering that vectors and vector quantities are a physics topic in which many students have difficulties, it is important to explore new ways to present them. In this vein, this study aims to explore a new m-learning application named LabVetor. The application is designed for teaching and learning vectors on mobile devices. LabVetor can either be used by teachers in the classroom, through interactive whiteboards, and by the students at home as an m-learning training application. The LabVetor was evaluated by a group of students and showed to be a useful tool to assist the comprehension and reflection of the content learned in classroom.
\end{abstract}

Resumo. O conhecimento e compreensão de vetores e grandezas vetoriais é extremamente importante para descrever o movimento dos objetos na física do ensino médio. Considerando-se que vetores e grandezas vetoriais são tópicos da física em que muitos estudantes têm dificuldades, é interessante explorar novas formas de apresentá-los. Nesse sentido, este trabalho tem como objetivo explorar uma nova aplicação de m-learning chamado LabVetor. $O$ aplicativo é projetado para ensino e aprendizagem de algumas operações simples com vetores em dispositivos móveis. LabVetor pode ser usado por professores em sala de aula, por meio de quadros interativos, e pelos alunos em casa, como uma aplicação m-learning para treinamento. O LabVetor foi avaliado por um grupo de estudantes e mostrou ser uma ferramenta útil para auxiliar a compreensão e reflexão do conteúdo apresentado em sala de aula.

\section{Introdução}

$\mathrm{Na}$ física do ensino médio há diversos tópicos que, longe de serem triviais e intuitivos, são de difícil compreensão para os alunos [Jimoyiannis and Komis, 2001]. No que tange ao estudo dos movimentos incluem-se, por exemplo, vetores e grandezas vetoriais. Nesses tópicos, muitos estudantes possuem dificuldades para dominar os conceitos e entender suas propriedades por métodos convencionais, o que torna difícil as suas aplicações na resolução de diversos problemas da mecânica. Visando mitigar essas dificuldades, diversas iniciativas baseadas na simulação de fenômenos físicos foram desenvolvidas. Estas 


\section{CBIE-LACLO 2015}

Anais do XXVI Simpósio Brasileiro de Informática na Educação (SBIE 2015)

abordagens enriqueceram e atualizaram as atividades de ensino, bem como expandiram as possibilidades de lecionar [Silva, 2011], na tentativa de aumentar o interesse dos alunos pelo conteúdo ensinado na física, tornando seu entendimento mais agradável [Filho, 2011].

Uma das iniciativas para melhorar a qualidade de ensino e aprendizagem dos conteúdos da física é o aprendizado por meio de dispositivos móveis (m-learning). $\mathrm{O}$ m-learning apresenta diversos pontos positivos, sendo um deles o fato de já fazer parte do cotidiano das pessoas em geral. Assim, eles podem ser usados como um complemento às salas de aula. Ademais, a geração mais nova é fortemente atraída pelo uso de dispositivos móveis [Toteja and Kumar, 2012]. Todas essas características justificam a grande aceitação de seu uso por parte de alunos e professores em ambientes educacionais [Abachi and Muhammad, 2014] e seu próspero crescimento.

Diante desse cenário, desenvolvemos o LabVetor, que é um aplicativo para dispositivos móveis que atua como uma ferramenta visando ao incremento dos recursos didáticos no ensino da Física e, em alguns casos, da Matemática. $\mathrm{O}$ aplicativo tem por objetivo ser uma ferramenta para auxiliar no ensino dos conceitos de vetores e grandezas vetoriais de modo a concretizar um pouco da sua abstração ao simular suas propriedades.

O LabVetor é um aplicativo m-learning utilizado para apoiar o ensino e aprendizado de vetores tanto pelo professor em sala de aula quanto pelos alunos em casa. Em sala de aula, o LabVetor é um laboratório virtual de ensino vetores projetado para uso em quadro interativo. Em casa, o aplicativo pode ser usado para apoiar o aprendizado do aluno ao fixar o conteúdo a partir de prática assistida. Esse último uso é de grande serventia, pois as principais informações de uso são transmitidas para um serviço web (web service) que armazena os erros e acertos dos alunos em um banco de dados. Essas informações podem ser usadas posteriormente para análise do aprendizado do aluno, fornecendo uma percepção mais detalhada do processo.

Além desta introdução, este trabalho é constituído por mais cinco seções. Na seção 2 são apresentados os conceitos gerais de vetores. A seção 3 apresenta os trabalhos relacionados. A seção 4 descreve a metodologia: o aplicativo e suas funcionalidades. Já a seção 5 apresenta a avaliação experimental realizada e os resultados obtidos. Por fim, na seção 6, são apresentadas as considerações finais.

\section{Ensino de Vetores na Física}

\subsection{Grandezas vetoriais}

As grandezas vetoriais são aquelas que possuem magnitude, direção e sentido, o que as diferencia das grandezas escalares que apenas se definem pela magnitude. Como um exemplo de grandezas vetoriais pode-se citar a velocidade, que possui uma direção e sentido, para onde a partícula está se deslocando, e a magnitude, o quão rápido o corpo se desloca nessa direção. Como exemplos de grandezas escalares, temos a massa, temperatura e área.

Entender e dominar os conceitos das grandezas vetoriais, suas propriedades e operações são importantes para compreender os tópicos de mecânica, pois o estudo e a análise do deslocamento, repouso, movimentos e ações de forças são diretamente ligadas aos vetores, uma vez que a velocidade, aceleração e a força são grandezas vetoriais. 


\section{CBIE-LACLO 2015}

Anais do XXVI Simpósio Brasileiro de Informática na Educação (SBIE 2015)

\subsection{Plataformas de Aprendizado}

Existe um conjunto grande de plataformas de aprendizado. No contexto relacionado ao presente trabalho, podem-se destacar os quadros interativos, os laboratórios virtuais e os ambientes de m-learning. Os quadros interativos são dispositivos eletrônicos que projetam imagens dos computadores em sua tela na qual o usuário pode interagir, sendo assim um dispositivo de entrada e saída de dados capaz de potencializar o espaço de ensino-aprendizagem [Melo and Ferreira, 2014]. É particularmente interessante quando associados a aprendizado baseado em inteligência coletiva.

Os laboratórios virtuais são aplicativos que tem o objetivo de simular um experimento de laboratório ou propriedades da natureza com fins educacionais. Experimentos e demonstrações de certas propriedades, que não poderiam ser vistos facilmente por estudantes pela ausência de laboratórios nas instituições, podem ser vistas usando simulações em computadores de mesa e dispositivos móveis. As simulações de experimentos são importantes na educação. As atividades experimentais, além de tornar o aprendizado mais interessante, são fundamentais no ensino das ciências por trazerem diversos benefícios na forma como ocorre a construção do conhecimento [Lucena et al., 2013].

A popularização e o fácil acesso aos dispositivos móveis, juntamente com os avanços em Tecnologias de Informação e Comunicação (TIC), ampliam a gama de possibilidades que podem ser utilizados para auxiliar a aprendizagem e os estudos de alunos [Voss et al., 2013]. Neste contexto, há diversos dispositivos móveis que permitem o uso de comunicação de dados e aplicações. Esses dispositivos e essa nova forma de interação possuem a capacidade de modificar a relação professor-aluno dentro e fora das salas de aulas, uma vez que esses aplicativos podem alterar as relações tanto no tempo (síncrona e assíncrona) [Hrastinski, 2008] quanto na distância (presencial e a distância) [Nortvig, 2014].

O m-learning pode ser entendido como uma parte específica do aprendizado eletrônico (e-learning) para dispositivos móveis [Georgiev et al., 2004]. O surgimento do m-learning foi viabilizado a partir da sofisticação dos dispositivos móveis que hoje possuem poder de processamento semelhante aos dos computadores de mesa, além de possuir recursos embutidos úteis para ferramentas educacionais, como, por exemplo, câmeras, GPS, leitores de mídia e tela sensível ao toque. Assim, juntando esses recursos, surgem iniciativas que proporcionam a estudantes e professores plataformas de maior interação [Mühlbeier et al., 2012].

\section{Trabalhos relacionados}

Tendo em vista esses princípios, foi conduzida uma busca sistemática tanto no ScienceDirect quanto no IEEExplorer usando a string ("physics" $\wedge$ "high school" $\wedge$ "m-learning") que retornaram, respectivamente, 49 e 38 resultados. Em relação aos resultados, poucos que eram referentes diretos à m-learning e ensino da física. Diante dos poucos resultantes encontrados, separam-se os trabalhos em dois grupos. No primeiro grupo, são incluídos os trabalhos feitos para auxiliar no ensino geral da física em aplicações m-learning. No segundo grupo, encontram-se trabalhos relacionados ao domínio de vetores e grandezas vetoriais.

No primeiro grupo, encontram-se trabalhos [de Marcos et al., 2010; Huesca et al., 2009] que fazem uso de aplicação mobile ou web com a função de permitir acesso dos 


\section{CBIE-LACLO 2015}

Anais do XXVI Simpósio Brasileiro de Informática na Educação (SBIE 2015)

alunos a um servidor onde encontram-se diversos questionários. Seu uso é feito tanto por professores, para elaborar o questionário, quanto por alunos para responder os questionários disponíveis com o propósito de ser um treinamento online e medir o desempenho dos alunos nas atividades propostas. Estes trabalhos se assemelham ao LabVetor uma vez que um dos objetivos da aplicação é armazenar as informações de uso em um servidor para medir o desempenho dos alunos.

O segundo grupo contempla os trabalhos mais diretamente relacionados ao domínio. Destaca-se o trabalho Vector Operation [Mozzaquatro et al., 2014], que mais se assemelha a este trabalho na questão do tema vetor. Apesar do Vector Operation também ter sido desenvolvido usando o MIT AppInventor 2 e ter a mesma temática de vetores, o seu propósito difere do LabVetor. O Vector Operation é como uma calculadora de vetores para auxiliar no estudo da física, onde são apenas inseridos os valores para as coordenadas dos vetores e o resultado da operação, soma ou multiplicação, é revelado ao aluno. No LabVetor, os vetores são representados graficamente e são "desenhados" pelo aluno, concretizando parte de sua abstração. Ademais, o propósito do LabVetor é ajudar no ensino e aprendizagem das operações com vetores, facilitando a visualização e construção do processo, enquanto o Vector Operation tem como finalidade ser uma ferramenta para ajudar a resolver problemas e questões com vetores.

Considerando os trabalhos citados e suas propostas, pode-se observar que não foram encontrados trabalhos com o mesmo propósito do LabVetor, o que nos permite dizer que o LabVetor é uma aplicação nova e original que combina os conceitos de laboratórios virtuais com m-learning visando a uma melhor experiência do aprendizado de vetores na Física do ensino médio.

\section{Aplicativo LabVetor}

Ao serem apresentados aos vetores, os alunos do ensino médio confundem, com grande frequência, operações de soma e subtração de vetores com as operações com números reais que já lhe são familiares. O objetivo principal do LabVetor é usar o dispositivo móvel para criar um cenário em que o processo gráfico de operar com vetores possa se tornar mais intuitivo. Assim, equações vetoriais são geradas aleatoriamente, ou formuladas por algum instrutor, e o aluno as resolve graficamente, por meio da interação via toque em um plano quadriculado. O seu uso se assemelha ao de um quadro interativo para dispositivos móveis, no qual o aluno "desenha" os vetores como se estivesse desenhando em um quadro.

Durante todo uso, a aplicação também faz o levantamento do desempenho do aluno ao resolver as equações. Esse registro permite que tanto o professor quanto os alunos possam acompanhar a evolução do processo de ensino e aprendizado.

\subsection{Arquitetura e Modelagem}

O aplicativo tem uma arquitetura voltada a apoiar dois modos gerais de operação: professor e aprendizado. A arquitetura pode ser detalhada na Figura 1.a. No modo professor, o aplicativo pode ser associado a um quadro interativo que permite o desenho dos vetores em uma tela quadriculada. No modo aprendizado, o aplicativo pode ser utilizado por alunos que tanto podem repassar o conteúdo ensinado quanto operar em modo prática. Em ambos os casos, o aplicativo transmite o conteúdo utilizado (questões, erros e acertos) para um serviço web. 


\section{CBIE-LACLO 2015}

Anais do XXVI Simpósio Brasileiro de Informática na Educação (SBIE 2015)

O diagrama de classes indicado na Figura 1.b apresenta as principais classes associadas ao problema. O usuário, seja professor ou aluno, acessa a tela do ambiente móvel por meio de uma interface baseada em quadros quadriculados (Figura 2) decompondo a tela em unidades discretas. As operações escalares de vetores e operações vetoriais, como soma e subtração, também são elementos que fazem parte das equações modeladas pelo aplicativo. As equações são instanciadas aleatoriamente.
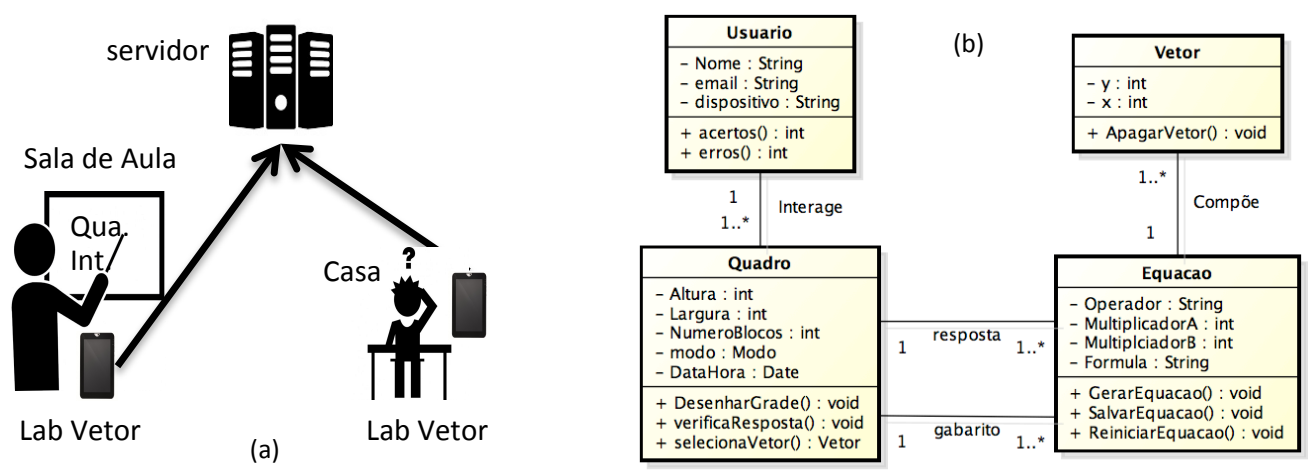

Figura 1. Arquitetura do LabVetor (a), Diagrama de Classes (b)

\subsection{Desenvolvimento}

O LabVetor foi desenvolvido para Android utilizando o MIT AppInventor 2, uma ferramenta de programação baseada em programação visual de blocos. Essa característica a torna de fácil uso para novatos em programação, mas também serve como um meio de desenvolver provas de conceito [Gomes and de Melo, 2013], poupando tempo dos programadores na fase de prototipagem [AppInventor, 2015]. O LabVetor foi desenvolvido buscando a facilidade de uso na operação com vetores, de tal forma que as instruções são passadas por meio de uma voz sintetizada oriunda do Text-To-Speech do Android, que diz o que o aluno deve fazer em cada tela.

A aplicação é composta por quatro telas, de modo que a tela inicial é um menu que leva às demais telas. As telas seguintes tem maneiras semelhantes de uso e dividem-se nos seguintes modos: ensino, prática e professor. O modo ensino contém textos explicativos de como utilizar os recursos do aplicativo. Ela contempla a função de desenhar e modificar vetores no quadro quadriculado, explicando aos alunos os conceitos básicos de vetores e grandezas vetoriais, como por exemplo, vetores equivalentes, multiplicação de vetores e operações vetoriais. Esses conceitos são fundamentais para que o aluno possa utilizar o LabVetor no modo prática.

O modo prática é o modo principal da aplicação. A partir dele, levantam-se os dados que possibilitam a avaliação de desempenho dos alunos. Conforme apresentado na Figura 2, o objetivo deste modo é que o aluno represente as equações apresentadas no quadro da direita, baseando-se nos vetores indicados no quadro da esquerda. Neste modo, a equação é gerada aleatoriamente, i.e., os multiplicadores de A e B podem variar de -2 a 2 (com exceção do zero), o operador da equação pode ser uma adição (+) ou subtração (-). A direção e sentido dos vetores também são gerados aleatoriamente. O seu comprimento pode variar de zero até quatro unidades. No quadro da direita, o aluno desenha os vetores segundo a operação solicitada. Para isso, ele deve pressionar o botão relativo ao vetor 


\section{CBIE-LACLO 2015}

Anais do XXVI Simpósio Brasileiro de Informática na Educação (SBIE 2015)

que deseja manipular e, na sequencia, pressionar o quadro para que o ponteiro de vetor torne-se visível. $\mathrm{O}$ aluno pode arrastar suas extremidades e moldar o vetor como proposto pela equação. Ao desenhar os três vetores no quadro, o aplicativo libera o botão verificar, que diz ao aluno os seus acertos e erros. No caso de presença de erro, o botão solução se torna disponível. A partir dele, pode-se exibir a resolução correta e as possíveis causas que levaram o aluno ao erro. Todos os dados de cada exercício praticado são enviados para o servidor.

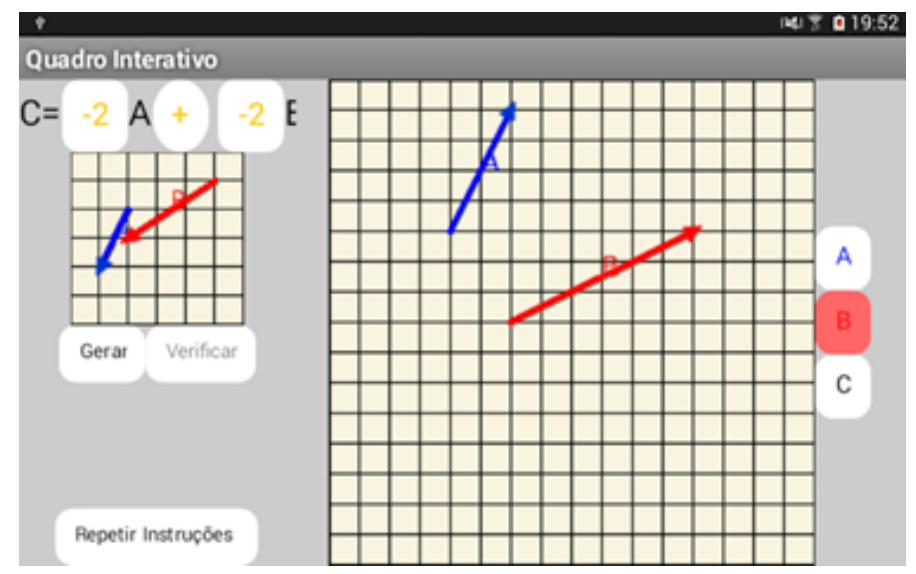

Figura 2. Interface do LabVetor

O modo professor é similar ao modo ensino, com exceção de que este permite que o professor modifique a equação, alterando os multiplicadores de A e B e o operador. Os vetores dados também são especificados no modo professor, ao invés de serem gerados aleatoriamente, como no modo prática.

\section{Avaliação Experimental}

A avaliação experimental foi baseada na utilização do aplicativo por voluntários. Os voluntários receberam instruções disponibilizadas no site do SourceForge ${ }^{1}$, dentre as quais baixar o aplicativo LabVetor em seus dispositivos móveis a partir do Google Play Store. O roteiro de avaliação, formulário de avaliação e resultados experimentais estão disponíveis no site do SourceForge.

Participaram da avaliação 101 voluntários que foram classificados em alunos (67) e indicações via internet (34). Os resultados experimentais foram obtidos tanto pelo uso direto do aplicativo LabVetor quanto pela resposta do formulário de avaliação. Conforme pode ser observado pela Figura 3.a, a faixa etária dos voluntários foi de 15-51 anos, sendo que a parcela de 10-20 anos corresponde a maioria dos voluntários.

A categorização dos voluntários foi feita segundo o nível de escolaridade e o conhecimento prévio acerca de vetores. A Figura 3.b apresenta a classificação dos voluntários quanto ao nível de escolaridade: (i) fundamental (2), (ii) $1^{\mathrm{a}}$ ano do ensino médio (57), (iii) $2^{\mathrm{a}}$ ano do ensino médio (24), (iii) $3^{\mathrm{a}}$ ano do ensino médio (8) e (iv) nível superior (10). A Figura 3.c indica o conhecimento prévio dos voluntários acerca de vetores e a grandezas vetoriais. A quantidade de voluntários que já conheciam e desconheciam

\footnotetext{
${ }^{1}$ https://sourceforge.net/p/gpca/wiki/labvetor/
} 


\section{CBIE-LACLO 2015}

Anais do XXVI Simpósio Brasileiro de Informática na Educação (SBIE 2015)

o assunto era, respectivamente, 39 e 61 , sendo que grande parte dos que desconheciam eram do $1^{\mathrm{a}}$ ano do ensino médio.

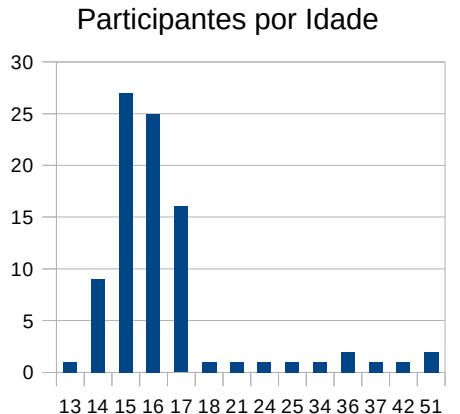

(a)

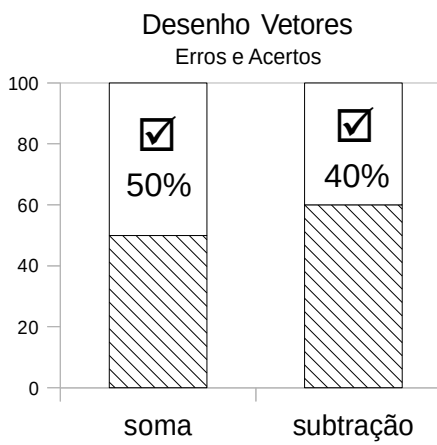

(d)

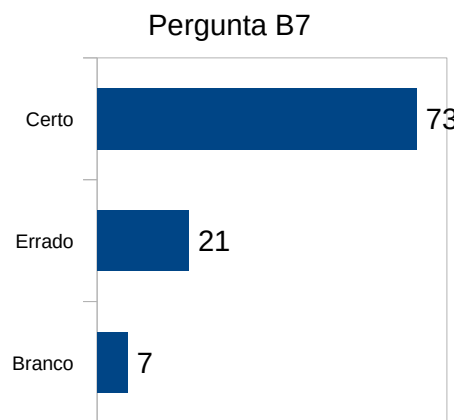

(g)

Avaliação do Aplicativo

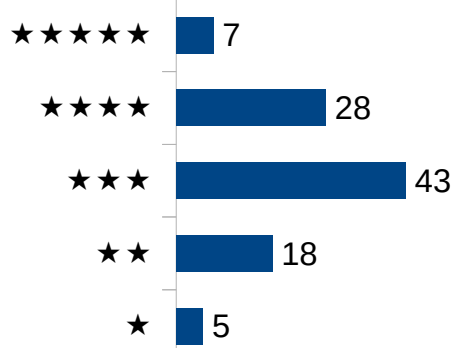

(j)

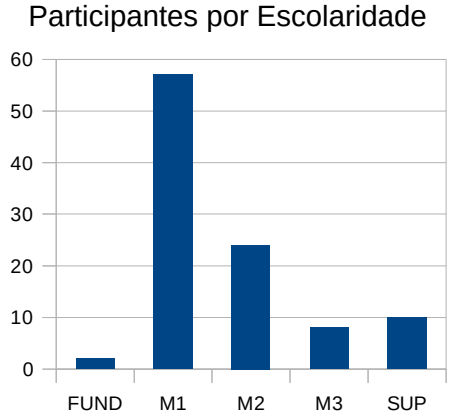

(b)

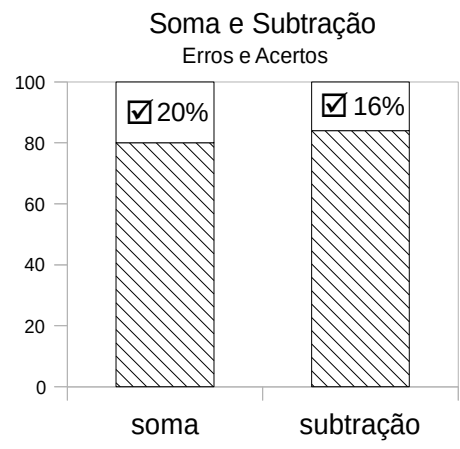

(e)

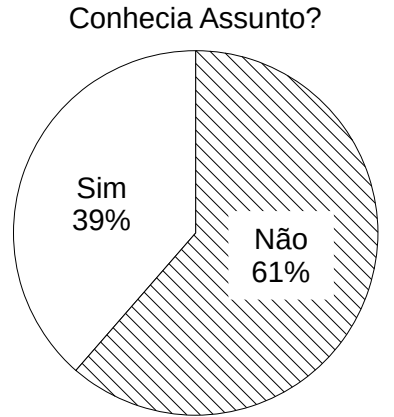

(c)

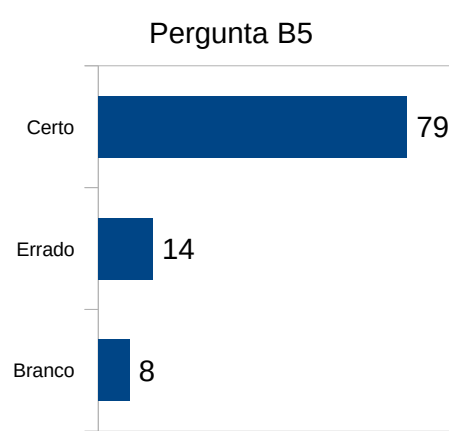

(f)

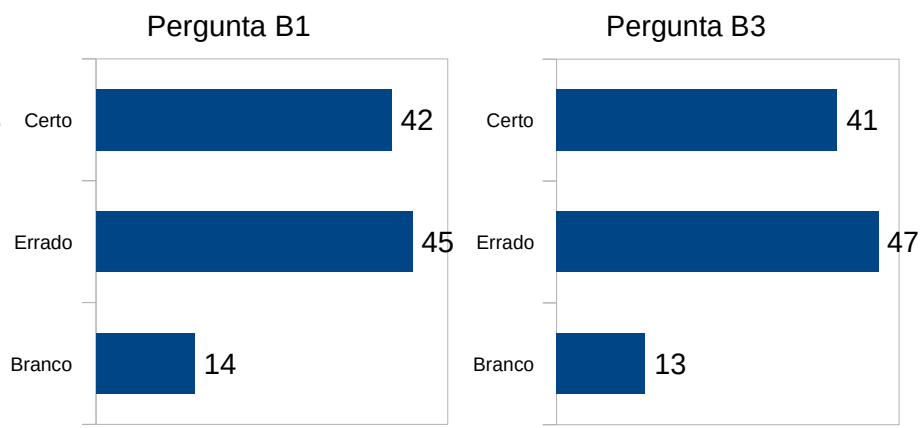

(h)

(i)

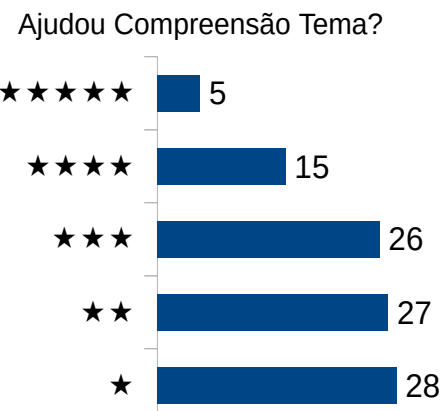

(k)

Defeitos Encontrados

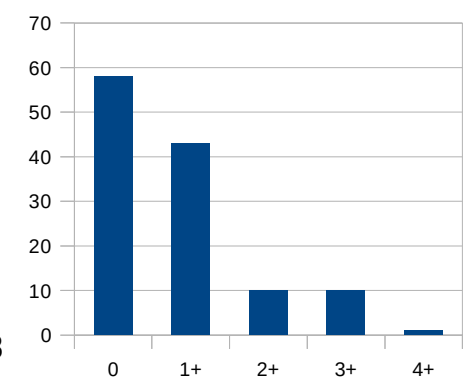

(I)

Figura 3. Avaliação Experimental

A partir dos dados produzidos diretamente pelo LabVetor e coletados via webser- 


\section{CBIE-LACLO 2015}

Anais do XXVI Simpósio Brasileiro de Informática na Educação (SBIE 2015)

vice, pôde-se identificar os erros e acertos dos exercícios de produto escalar e dos resultados das operações de soma e subtração de vetores. Os exercícios do LabVetor exploram uma expressão $\mathrm{C}=\mathrm{aA} \pm \mathrm{bB}$, onde as constantes a e $\mathrm{b}$ e os vetores $\mathrm{A}$ e $\mathrm{B}$ são apresentados no enunciado. $\mathrm{O}$ usuário deve desenhar os vetores aA e bB e, finalmente, produzir o vetor $\mathrm{C}$ a partir da soma ou diferença entre os vetores.

A Figura 3.d apresenta a quantidade de erros e acertos ao desenhar os vetores aA e bB objetivando avaliar o desempenho dos voluntários nas operações de produtos escalares. A partir da Figura 3.d, observa-se um erro nos produtos escalares de $50 \%$ e $60 \%$ nas operações de soma e subtração, respectivamente. Já a Figura 3.e apresenta a quantidade de erros e acertos ao desenhar o vetor resultante $\mathrm{C}$, objetivando avaliar o desempenho dos voluntários nas operações de soma e subtração propriamente dita. Observa-se um erro de $80 \%$ e $84 \%$ nas operações de soma e subtração, respectivamente.

Pode-se observar uma taxa elevada de erros tanto nas operações de produtos escalares quanto nas operações de somas e subtrações propriamente ditas. Em um primeiro momento a quantidade de erros observados pode não ser um bom indicador, mas em se tratando de uma ferramenta de apoio ao estudo, a quantidade de erros serve como um indicador para alunos e professores sobre a necessidade de reforço do conteúdo estudado.

O uso do formulário de avaliação teve como objetivo analisar a reflexão dos voluntários acerca de vetores e grandezas vetoriais de um modo a transcender o aprendizado mecânico. Podem-se destacar as seguintes perguntas constantes no formulário:

- B5: Dois vetores com o mesmo módulo são sempre considerados iguais?

- B7: Dois vetores com o mesmo módulo, direção e sentido são sempre considerados iguais?

- B1: Em uma soma entre vetores, o vetor resultante tem sempre módulo maior do que a soma dos módulos de suas parcelas?

- B3: Em uma multiplicação de um vetor por um número escalar, o vetor resultante é sempre maior que o vetor original?

As perguntas B5 e B7 tinham como foco a compreensão do sentido e módulo dos vetores. A Figura 3.f apresenta a síntese das respostas para a pergunta B5. Esperava-se que o usuário respondesse não à pergunta. A quantidade de voluntários que respondeu corretamente foi significativamente superior aos que erraram e aos que não souberam responder. A síntese das respostas a pergunta B7 está disponível na Figura 3.g. Esperavase que o usuário respondesse sim à pergunta. A quantidade de alunos que respondeu corretamente foi significativamente superior aos que erraram e aos que não souberam responder.

As perguntas B1 e B3 tinham como foco a percepção de algumas propriedades das operações de soma e produto escalar. É importante ressaltar que o aplicativo LabVetor apresenta as operações, mas não explora a reflexão dessas propriedades. A Figura 3.h apresenta a síntese das respostas para a pergunta B1. Da mesma forma, a síntese das respostas da pergunta B3 está disponível na Figura 3.i. Esperava-se que o usuário respondesse não a ambas as perguntas. A quantidade de voluntários que respondeu corretamente foi inferior aos que erraram.

A seguir, foi feita uma sequência de análises em cima do LabVetor propriamente dito. A Figura 3.j apresenta a percepção do usuário sobre o aplicativo, avaliando-o em 


\section{CBIE-LACLO 2015}

Anais do XXVI Simpósio Brasileiro de Informática na Educação (SBIE 2015)

uma escala de 1 (muito ruim) a 5 (muito bom). Foi realizado um teste estatístico de uma amostra Wilcoxon visando verificar se a distribuição é simétrica à 3 (regular). $\mathrm{O} p$-value encontrado foi $16.1 \%$, indicando que não há evidência para refutar a hipótese nula, i.e., o aplicativo teve desempenho igual a 3 (regular). O segundo teste, indicado pela Figura 3.k, teve por objetivo avaliar, em uma escala de 1 a 5 , se o voluntário considera que o LabVetor o ajudou a compreender a temática de vetores e grandeza vetoriais. Neste caso, observou-se que a grande parte dos voluntários indicou não ter aprendido diretamente com o aplicativo. Este resultado é relevante uma vez que a maioria dos voluntários não dominava o tema. Ademais, o resultado justifica a importância da versão voltada ao quadro interativo para ser utilizada pelo professor durante a aula. Realizou-se o teste estatístico Wilcoxon e obteve-se um p-value de menos de $0.01 \%$ que refutou a hipótese nula, levando a hipótese alternativa de que a distribuição é inferior à 3 (regular).

O terceiro e quarto testes, indicaram, respectivamente, o número de defeitos observados e um teste de usabilidade SUS [Albert and Tullis, 2013], constituídos pelas perguntas E1 a E10 do formulário de avaliação. Em se tratando de defeitos, a maioria dos usuários percebeu um defeito indicado na Figura 3.1. Um grupo menor observou 2 defeitos e uma parcela pequena de voluntários observou de 3 a 4 defeitos durante o uso do aplicativo, o que indica que há uma necessidade de maior depuração do aplicativo. Finalmente, com relação ao teste SUS, foi observada uma pontuação igual a 51. Um aplicativo é considerado acima da média se possuir uma pontuação acima de 68. Neste caso, o LabVetor apresentou um desempenho abaixo da média, indicando que há espaço para melhorias neste quesito.

\section{Conclusão}

Exploramos, com o LabVetor, uma aplicação m-learning projetada para ensino e aprendizagem de operações com vetores em dispositivos móveis. O LabVetor pode ser usado tanto por professores em sala de aula, por meio de quadros interativos, quanto pelos alunos em casa, como uma aplicação para treinamento. O LabVetor foi avaliado por um grupo de voluntários com diferentes perfis e mostrou ser uma ferramenta útil para auxiliar a compreensão e reflexão do conteúdo aprendido em sala de aula. As avaliações apresentadas neste artigo corroboram para a necessidade do caráter dual da aplicação (modo professor e aluno), uma vez que quando usado apenas no seu modo aluno (por alunos que desconheciam o assunto), o aplicativo não trouxe resultados satisfatórios. Finalmente, apesar do seu aspecto inovador, os resultados em termos de usabilidade apontam a necessidade de um maior aprimoramento da ferramenta neste quesito.

\section{Agradecimentos}

Os autores agradecem à FAPERJ e ao CNPq pelo financiamento parcial do trabalho.

\section{Referências}

Abachi, H. R. and Muhammad, G. (2014). The impact of m-learning technology on students and educators. Computers in Human Behavior, 30:491-496.

Albert, W. and Tullis, T. (2013). Measuring the User Experience, Second Edition: Collecting, Analyzing, and Presenting Usability Metrics. Morgan Kaufmann, Amsterdam ; Boston, 2 edition edition. 
AppInventor, M. (2015). MIT App Inventor. Technical report, http://appinventor.mit.edu/explore/.

de Marcos, L., Hilera, J. R., Barchino, R., Jiménez, L., Martínez, J. J., Gutiérrez, J. A., Gutiérrez, J. M., and Otón, S. (2010). An experiment for improving students performance in secondary and tertiary education by means of m-learning auto-assessment. Computers \& Education, 55(3):1069-1079.

Filho, J. V. d. S. (2011). Utilizando Ambientes Virtuais como ferramenta de auxílio no ensino de Física em escolas públicas. In Anais do Simpósio Brasileiro de Informática na Educação, volume 1.

Georgiev, T., Georgieva, E., and Smrikarov, A. (2004). M-learning: A New Stage of e-Learning. In Proceedings of the 5th International Conference on Computer Systems and Technologies, CompSysTech '04, pages 1-5, New York, NY, USA. ACM.

Gomes, T. C. and de Melo, J. C. (2013). App Inventor for Android: Uma Nova Possibilidade para o Ensino de Lógica de Programação. In Anais dos Workshops do Congresso Brasileiro de Informática na Educação, volume 2.

Hrastinski, S. (2008). Asynchronous and synchronous e-learning. Educause quarterly, 31(4):51-55.

Huesca, G., Robledo-Rella, V., Noguez, J., and Neri, L. (2009). Aaprender: Combining on-line training and virtual learning environments to improve problem solving skills. In 39th IEEE Frontiers in Education Conference, 2009. FIE '09, pages 1-6.

Jimoyiannis, A. and Komis, V. (2001). Computer simulations in physics teaching and learning: a case study on students' understanding of trajectory motion. Computers \& Education, 36(2):183-204.

Lucena, G. L., dos Santos, V. D., and da Silva, A. G. (2013). Laboratório virtual como alternativa didática para auxiliar o ensino de química no ensino médio. Revista Brasileira de Informática na Educação, 21(02):27.

Melo, P. C. d. O. and Ferreira, V. G. G. (2014). A Lousa Digital no ensino de Matemática: análise das interações docentes. Revista Brasileira de Informática na Educação, 22(02): 109.

Mozzaquatro, P. M., Paschoal, L. N., Figueiró, M. F., Kronbauer, F. S., and Antoniazzi, R. L. (2014). Software Educativo para o ensino de vetores integrado aos conceitos de Cloud Computing e M-Learning. Revista Eletrônica Argentina-Brasil de Tecnologias da Informação e da Comunicação, 1(1).

Mühlbeier, A. R. K., Mozzaquatro, P. M., Medina, R. D., Oliveira, L. C. d., Moreira, R. C., and Antoniazzi, R. L. (2012). MOBILE HQ: O Uso de Softwares Educativos na Modalidade M-Learning. Anais do Simpósio Brasileiro de Informática na Educação, 23(1).

Nortvig, M. (2014). The Change of Time and Space in E-Learning. American Journal of Educational Research, 2(8):612-616.

Silva, S. (2011). Desenvolvimento de um laboratório virtual para ensino de física em cursos de engenharia através de physlets. In Congresso Brasileiro de Edudação em Engenharia.

Toteja, R. and Kumar, S. (2012). Usefulness of M-Devices in Education: A Survey. Procedia - Social and Behavioral Sciences, 67:538-544.

Voss, G. B., Nunes, F. B., Herpich, F., and Medina, R. D. (2013). Ambientes Virtuais de Aprendizagem e Ambientes Imersivos: um estudo de caso utilizando tecnologias de computação móvel. Anais do Simpósio Brasileiro de Informática na Educação, 24(1). 